\title{
Classification of Pulse Waveforms Using Edit Distance with Real Penalty
}

\author{
Dongyu Zhang, ${ }^{1}$ Wangmeng Zuo, ${ }^{1}$ David Zhang,, ${ }^{1,2}$ Hongzhi Zhang, ${ }^{1}$ and Naimin Li1 \\ ${ }^{1}$ Biocomputing Research Centre, School of Computer Science and Technology, Harbin Institute of Technology, Harbin, 150001, China \\ ${ }^{2}$ Shenzhen Graduate School, Harbin Institute of Technology, Shenzhen 518055, China
}

Correspondence should be addressed to Wangmeng Zuo, cswmzuo@gmail.com

Received 13 March 2010; Revised 12 June 2010; Accepted 25 August 2010

Academic Editor: Christophoros Nikou

Copyright ( 2010 Dongyu Zhang et al. This is an open access article distributed under the Creative Commons Attribution License, which permits unrestricted use, distribution, and reproduction in any medium, provided the original work is properly cited.

\begin{abstract}
Advances in sensor and signal processing techniques have provided effective tools for quantitative research in traditional Chinese pulse diagnosis (TCPD). Because of the inevitable intraclass variation of pulse patterns, the automatic classification of pulse waveforms has remained a difficult problem. In this paper, by referring to the edit distance with real penalty (ERP) and the recent progress in $k$-nearest neighbors $(\mathrm{KNN})$ classifiers, we propose two novel ERP-based KNN classifiers. Taking advantage of the metric property of ERP, we first develop an ERP-induced inner product and a Gaussian ERP kernel, then embed them into difference-weighted KNN classifiers, and finally develop two novel classifiers for pulse waveform classification. The experimental results show that the proposed classifiers are effective for accurate classification of pulse waveform.
\end{abstract}

\section{Introduction}

Traditional Chinese pulse diagnosis (TCPD) is a convenient, noninvasive, and effective diagnostic method that has been widely used in traditional Chinese medicine (TCM) [1]. In TCPD, practitioners feel for the fluctuations in the radial pulse at the styloid processes of the wrist and classify them into the distinct patterns which are related to various syndromes and diseases in TCM. This is a skill which requires considerable training and experience, and may produce significant variation in diagnosis results for different practitioners. So in recent years techniques developed for measuring, processing, and analyzing the physiological signals $[2,3]$ have been considered in quantitative TCPD research as a way to improve the reliability and consistency of diagnoses [4-6]. Since then, much progress has been made: a range of pulse signal acquisition systems have been developed for various pulse analysis tasks [7-9]; a number of signal preprocessing and analysis methods have been proposed in pulse signal denoising, baseline rectification [10], segmentation [11]; many pulse feature extraction approaches have been suggested by using various timefrequency analysis techniques [12-14]; many classification methods have been studied for pulse diagnosis $[15,16]$ and pulse waveform classification [17-19].

Pulse waveform classification aims to assigning a traditional pulse pattern to a pulse waveform according to its shape, regularity, force, and rhythm [1]. However, because of the complicated intra-class variation in pulse patterns and the inevitable influence of local time shifting in pulse waveforms, it has remained a difficult problem for automatic pulse waveform classification. Although researchers have developed several pulse waveform classification methods such as artificial neural network $[18,20,21]$, decision tree [22], and wavelet network [23], most of them are only tested on small data sets and usually cannot achieve satisfactory classification accuracy.

Recently, various time series matching methods, for example, dynamical time warping (DTW) [24] and edit distance with real penalty (ERP) [25], have been applied for time series classification. Motivated by the success of time series matching techniques, we suggest utilizing time series classification approaches for addressing the intraclass variation and the local time shifting problems in pulse waveform classification. In this paper, we first develop an ERP-induced inner product and a Gaussian ERP (GERP) 


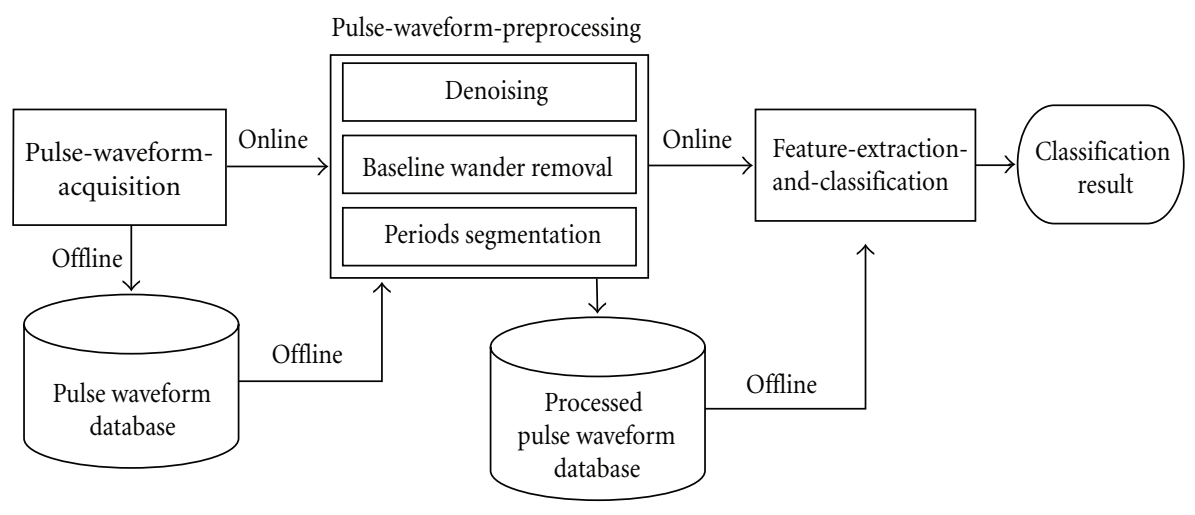

FIgURE 1: Schematic diagram of the pulse waveform classification modules.

kernel function. Then, with the difference-weighted KNN (DFWKNN) framework [26], we further present two novel ERP-based classifiers: the ERP-based difference-weighted KNN classifier (EDKC) and the kernel difference-weighted KNN with Gaussian ERP kernel classifier (GEKC). Finally, we evaluate the proposed methods on a pulse waveform data set of five common pulse patterns, moderate, smooth, taut, unsmooth, and hollow. This data set includes 2470 pulse waveforms, which is the largest data set used for pulse waveform classification to the best of our knowledge. Experimental results show that the proposed methods achieve an average classification rate of $91.74 \%$, which is higher than those of several state-of-the-art approaches.

The remainder of this paper is organized as follows. Section 2 introduces the main modules in pulse waveform classification. Section 3 first presents a brief survey on ERP and DFWKNN, and then proposes two novel ERPbased classifiers. Section 4 provides the experimental results. Finally, Section 5 concludes this paper.

\section{The Pulse Waveform Classification Modules}

Pulse waveform classification usually involves three modules: a pulse waveform acquisition module, a preprocessing module, and a feature extraction and classification module. The pulse waveform acquisition module is used to acquire pulse waveforms with satisfactory quality for further processing. The preprocessing module is used to remove the distortions of the pulse waveforms caused by noise and baseline wander. Finally, using the feature extraction and classification module, pulse waveforms are classified into different patterns (Figure 1).

2.1. Pulse Waveform Acquisition. Our pulse waveform acquisition system is jointly developed by the Harbin Institute of Technology and the Hong Kong Polytechnic University. The system uses a motor-embedded pressure sensor, an amplifier, a USB interface, and a computer to acquire pulse waveforms. During the pulse waveform acquisition, the sensor (Figure 2(a)) is attached to wrist and contact pressure is applied by the computer-controlled automatic rotation of motors and mechanical screws. Pulse waveforms acquired by the pressure sensors are transmitted to the computer through the USB interface. Figure 2(b) shows an image of the scene of the pulse waveform collection.

2.2. Pulse Waveform Preprocessing. In the pulse-waveformpreprocessing, it is necessary to first remove the random noise and power line interference. Moreover, as shown in Figure 3(a), the baseline wander caused by factors such as respiration would also greatly distort the pulse signal. We use a Daubechies 4 wavelet transform to remove the noise by empirically comparing the performance of several wavelet functions and correct the baseline wander using a waveletbased cascaded adaptive filter previously developed by our group [10].

Pulse waveforms are quasiperiodic signals where one or a few periods are sufficient to classify a pulse shape. So we adopt an automatic method to locate the position of the onsets, split each multiperiods pulse waveform into several single periods, and select one of these periods as a sample of our pulse waveform data set. Figure 3(b) shows the result of the baseline wander correction and the locations of the onsets of a pulse waveform.

\subsection{Feature Extraction and Classification. TCPD recognizes} more than 20 kinds of pulse patterns which are defined according to criteria such as shape, position, regularity, force, and rhythm. Several of these are not settled issues in the TCPD field but we can say that there is general agreement that, according to the shape, there are five pulse patterns, namely, moderate, smooth, taut, hollow, and unsmooth. Figure 4 shows the typical waveforms of these five pulse patterns acquired by our pulse waveform acquisition system. All of these pulses can be defined according to the presence, absence, or strength of three types of waves or peaks: percussion (primary wave), tidal (secondary wave), and dicrotic (triplex wave), which are denoted by $\mathrm{P}, \mathrm{T}$, and $\mathrm{D}$, respectively, in Figure 4. A moderate pulse usually has all three types of peaks in one period, a smooth pulse has low dicrotic notch (DN) and unnoticeable tidal wave, a taut pulse frequently exhibits a high-tidal peak, an unsmooth pulse exhibits unnoticeable tidal or dicrotic wave, and a hollow 


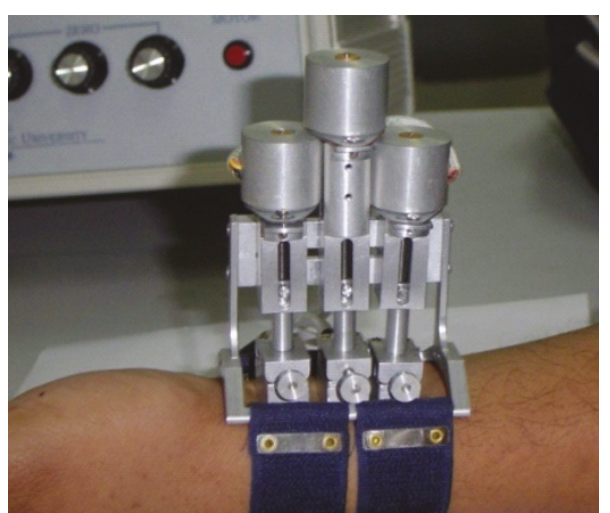

(a)

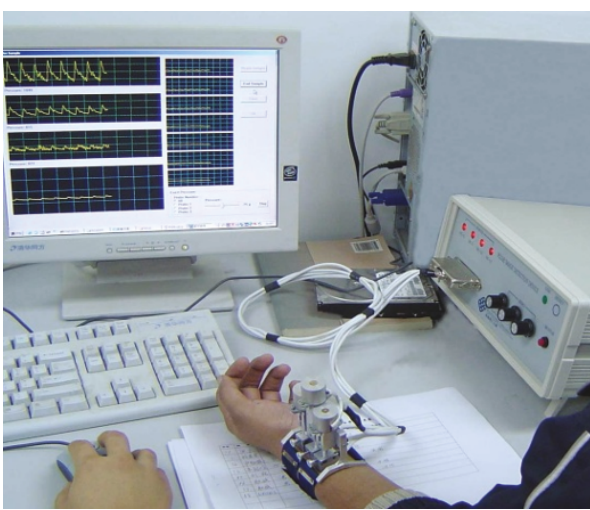

(b)

FIGURE 2: The pulse waveform acquisition system: (a) the motor embedded pressure sensor, and (b) the whole pulse waveform acquisition system.

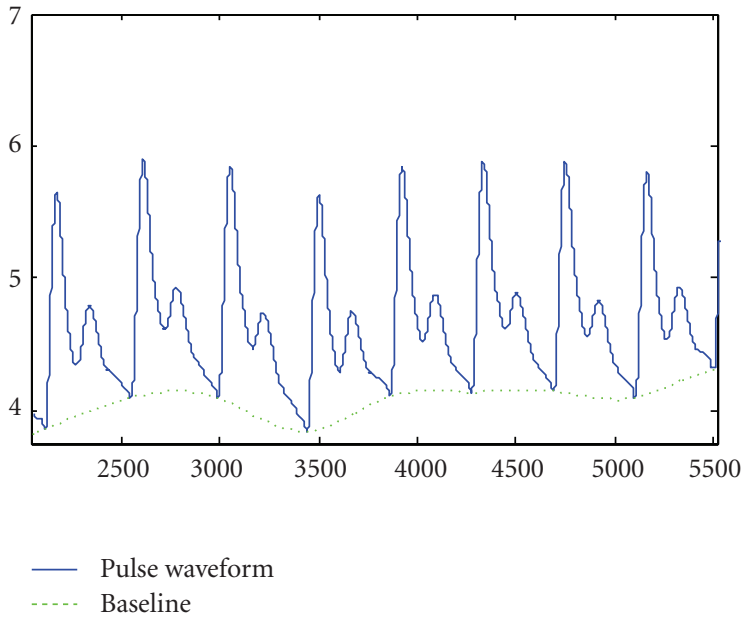

(a)

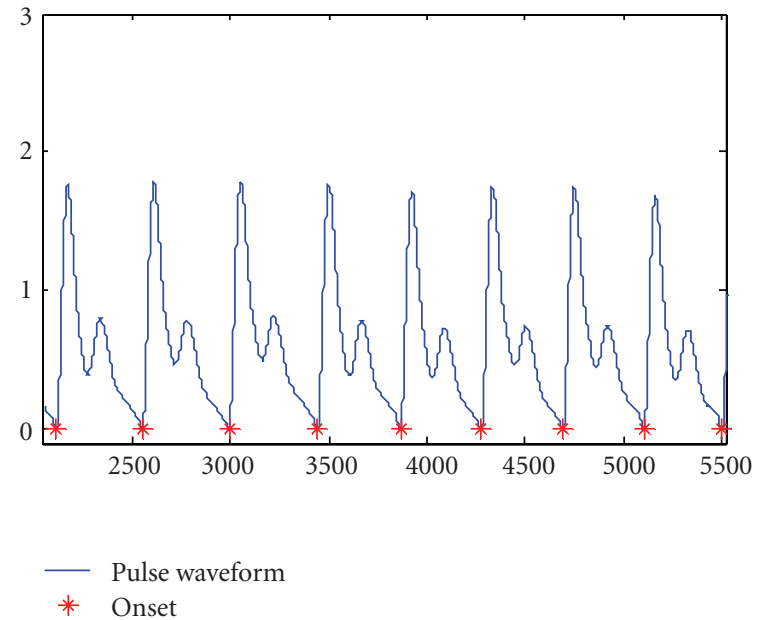

(b)

FIGURE 3: Pulse waveform baseline wander correction: (a) pulse waveform distorted by baseline wander, and (b) pulse waveform after baseline wander correction.

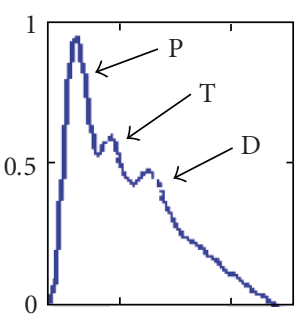

(a)

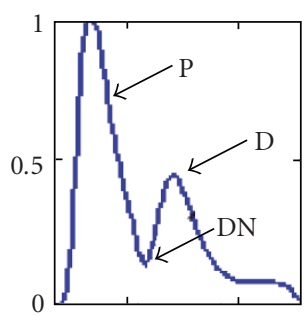

(b)

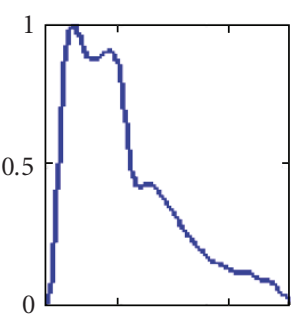

(c)

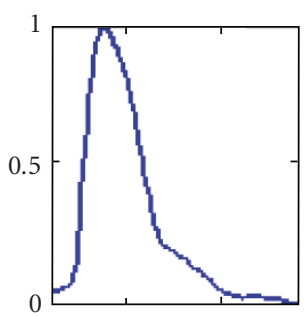

(d)

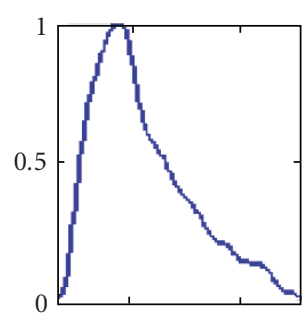

(e)

Figure 4: Five typical pulse patterns classified by shape: (a) moderate, (b) smooth, (c) taut, (d) hollow, and (e) unsmooth pulse patterns.

pulse has rapid descending part in percussion wave and unnoticeable dicrotic wave.

However, pulse waveform classification may suffer from the problems of small inter class and large intraclass variation. As shown in Figure 5, moderate pulse with unnoticeable tidal wave is similar to smooth pulse. For taut pulse, the tidal wave sometimes becomes very high or even merges with the percussion wave. Moreover, the factors such as local time axis distortion would make the classification problem more complicated.

So far, a number of pulse waveform classification approaches have been proposed, which can be grouped into 
two categories: the representation-based and the similarity measure-based methods. The representation-based methods first extract representative features of pulse waveforms using techniques such as spatiotemporal analysis [14], fast Fourier transform (FFT) [12], and wavelet transform [13]. Then the classification is performed in the feature space by using various classifiers, for example, decision tree [22] and neural network [18, 20, 21].

For the similarity measure-based methods, classification is performed in the original data space by using certain distance functions to measure the similarity of different pulse waveforms. Our pulse waveform classification approaches belong to the similarity measure-based method, where we first propose an ERP-induced inner product and a Gaussian ERP kernel, and then embed them into the DFWKNN and KDFWKNN classifiers [26, 27]. In the following section, we will introduce the proposed methods in detail.

\section{The EDCK and GEKC Classifiers}

In this section, we first provide a brief survey on related work, that is, ERP, DFWKNN, and KDFWKNN. Then we explain the basic ideas and implementations of the ERPbased DFWKNN classifier (EDKC) and the KDFWKNN with Gaussian ERP kernel classifier (GEKC).

3.1. Edit Distance with Real Penalty. The ERP distance is a state-of-the-art elastic distance measure for time series matching [25]. During the calculation of the ERP distance, two time series, $\mathbf{a}=\left[a_{1}, \ldots, a_{m}\right]$ with $m$ elements and $\mathbf{b}=$ $\left[b_{1}, \ldots, b_{n}\right]$ with $n$ elements, are aligned to the same length by adding some symbols (also called gaps) to them. Then each element in one time series is either matched to a gap or an element in the other time series. Finally the ERP distance between $\mathbf{a}$ and $\mathbf{b}, d_{\operatorname{erp}}(\mathbf{a}, \mathbf{b})$, is recursively defined as

$$
\begin{aligned}
& d_{\text {erp }}(\mathbf{a}, \mathbf{b}) \\
& =\left\{\begin{array}{ll}
\sum_{i=1}^{m}\left|a_{i}-g\right| & \text { if } n=0, \\
\sum_{i=1}^{n}\left|b_{i}-g\right| & \text { if } m=0, \\
d_{\operatorname{erp}}(\operatorname{Rest}(\mathbf{a}), \mathbf{b})+\left|a_{1}-g\right|, \\
d_{\operatorname{erp}}(\mathbf{a}, \operatorname{Rest}(\mathbf{b}))+\left|b_{1}-g\right|,
\end{array}\right\}, \text { otherwise, }
\end{aligned}
$$

where $\operatorname{Rest}(\mathbf{a})=\left[a_{2}, \ldots, a_{m}\right]$ and $\operatorname{Rest}(\mathbf{b})=\left[b_{2}, \ldots, b_{n}\right],|\cdot|$ denote the $l_{1}$-norm, and $g$ is a constant with a default value $g$ $=0$ [25]. From (1), one can see that the distance $d_{\operatorname{erp}}(\mathbf{a}, \mathbf{b})$ can be derived by recursively calculating the ERP distance of their subsequences until the length of one subsequence is zero.

By incorporating gaps in aligning time series of different length, the ERP distance is very effective in handling the local time shifting problem in time series matching. Besides, the ERP distance satisfies the triangle inequality and is a metric [25].
3.2. DFWKNN and KDFWKNN. DFWKNN and KDFWKNN are two recently developed KNN classifiers with classification performance comparable with or better than several state-of-the-art classification methods [26]. Let $X$ be a data set of $n$ samples $\left\{\mathbf{x}_{1}, \ldots, \mathbf{x}_{n}\right\}$, and the corresponding class labels are $\left\{y_{1}, \ldots, y_{n}\right\}$ with each element from $\left\{\omega_{j} \mid j \in[1, \ldots, c]\right\}$, where $c$ denotes the number of classes. For a test sample $\mathbf{x}$, its $k$-nearest neighbors from $X$ are found using the Euclidean distance to form a matrix $\mathbf{X}^{n n}=\left[\mathbf{x}_{1}^{n n}, \ldots, \mathbf{x}_{k}^{n n}\right]$. In DFWKNN, the weights of the $k$ nearest neighbors are defined as a vector $\mathbf{w}=\left[w_{1}, \ldots, w_{k}\right]^{\mathrm{T}}$, which can be obtained by solving the following constrained optimization problem:

$$
\begin{aligned}
& \mathbf{w}=\underset{\mathbf{w}}{\arg \min } \frac{1}{2}\left\|\mathbf{x}-\mathbf{X}^{n n} \mathbf{w}\right\|^{2} \\
& \text { subject to } \quad \sum_{i=1}^{k} w_{i}=1 .
\end{aligned}
$$

By defining the Gram matrix as

$$
\mathbf{G}=\left[\mathbf{x}-\mathbf{x}_{1}^{n n}, \ldots, \mathbf{x}-\mathbf{x}_{k}^{n n}\right]^{\mathrm{T}}\left[\mathbf{x}-\mathbf{x}_{1}^{n n}, \ldots, \mathbf{x}-\mathbf{x}_{k}^{n n}\right],
$$

the weight vector $\mathbf{w}$ can be obtained by solving $\mathbf{G w}=\mathbf{1}_{k}$, where $\mathbf{1}_{k}$ is a $k \times 1$ vector with all elements equal to 1 . If the matrix $\mathbf{G}$ is singular, there is no inverse of $\mathbf{G}$ and the solution of $\mathbf{w}$ would be not unique. To avoid this case, a regularization method is adopted by adding the multiplication of a small value with the identity matrix, and the weight vector $\mathbf{w}$ can be obtained by solving the system of linear equations:

$$
\left[\mathbf{G}+\frac{\eta \mathbf{I}_{k} \operatorname{tr}(\mathbf{G})}{k}\right] \mathbf{w}=\mathbf{1}_{k},
$$

where $\operatorname{tr}(\mathbf{G})$ is the trace of $\mathbf{G}, \eta \in\left[10^{-3} \sim 10^{0}\right]$ is the regularization parameter, $k$ is the number of nearest neighbors of $\mathbf{x}$, and $\mathbf{I}_{k}$ is a $k \times k$ identity matrix. Finally, using the weighted KNN rule, the class label $\omega_{j_{\max }}=$ $\arg \max _{\omega_{j}}\left(\sum_{y_{i}^{n n}=\omega_{j}} w_{i}\right)$ is assigned to the sample $\mathbf{x}$.

By defining the kernel Gram matrix, DFWKNN can be extended to KDFWKNN. Using the feature mapping $\mathbb{F}: \mathbf{x} \rightarrow$ $\phi(\mathbf{x})$ and the kernel function $\kappa\left(\mathbf{x}, \mathbf{x}^{\prime}\right)=\left\langle\phi(\mathbf{x}), \phi\left(\mathbf{x}^{\prime}\right)\right\rangle$, the kernel Gram matrix $\mathbf{G}^{\kappa}$ is defined as

$$
\begin{aligned}
\mathbf{G}^{\kappa}= & {\left[\phi(\mathbf{x})-\phi\left(\mathbf{x}_{1}^{n n}\right), \ldots, \phi(\mathbf{x})-\phi\left(\mathbf{x}_{1}^{n n}\right)\right]^{\mathrm{T}} } \\
& \times\left[\phi(\mathbf{x})-\phi\left(\mathbf{x}_{1}^{n n}\right), \ldots, \phi(\mathbf{x})-\phi\left(\mathbf{x}_{1}^{n n}\right)\right] .
\end{aligned}
$$

In KDFWKNN, the weight vector $\mathbf{w}$ is obtained by solving

$$
\left[\mathbf{G}^{\kappa}+\frac{\eta \mathbf{I}_{k} \operatorname{tr}\left(\mathbf{G}^{\kappa}\right)}{k}\right] \mathbf{w}=\mathbf{1}_{k} .
$$

For a detailed description of KDFWKNN, please refer to [26].

3.3. The EDKC Classifier. Current similarity measure-based methods usually adopt the simple nearest neighbor classifier. 


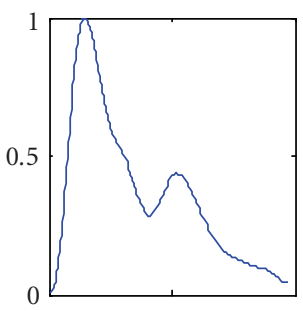

(a)

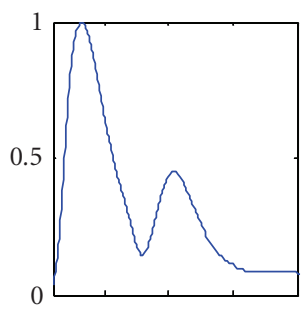

(b)

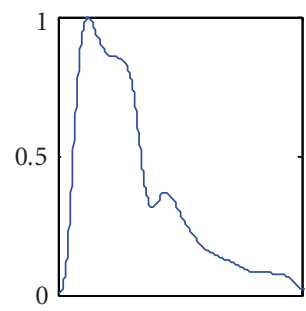

(c)

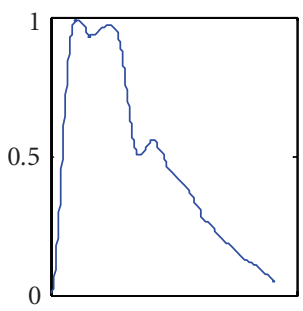

(d)

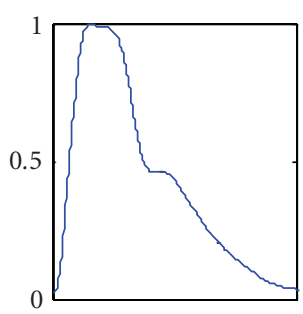

(e)

FIGURE 5: Inter- and intraclass variations of pulse patterns: (a) a moderate pulse with unnoticeable tidal wave is similar to (b) a smooth pulse; taut pulse patterns may exhibit different shapes, for example, (c) typical taut pulse, (d) taut pulse with high tidal wave, and (e) taut pulse with tidal wave merged with percussion wave.

Input: The unclassified sample $\mathbf{x}$, the training samples $X=\left\{\mathbf{x}_{1}, \ldots, \mathbf{x}_{n}\right\}$ with the corresponding class labels $\left\{y_{1}, \ldots, y_{n}\right\}$, the regularization parameter $\eta$, and the number of nearest neighbors $k$.

Output: The predicted class label $\omega_{j_{\max }}$ of the sample $\mathbf{x}$.

Step 1. Use the ERP distance to obtain the $k$-nearest neighbors of the sample $\mathbf{x}$,

$\mathbf{X}^{n n}=\left[\mathbf{x}_{1}^{n n}, \ldots, \mathbf{x}_{k}^{n n}\right]$, and their corresponding class labels $\left[y_{1}^{n n}, \ldots, y_{k}^{n n}\right]$.

Step 2. Calculate the ERP-induced inner product of the samples $\mathbf{x}$ and each of its nearest

neighbors, $\mathbf{k}_{\text {erp }}(i)=\left\langle\mathbf{x}, \mathbf{x}_{i}^{n n}\right\rangle_{\text {erp }}=\left(d_{\text {erp }}^{2}\left(\mathbf{x}, \mathbf{x}_{0}\right)+d_{\text {erp }}^{2}\left(\mathbf{x}_{i}^{n n}, \mathbf{x}_{0}\right)-d_{\text {erp }}^{2}\left(\mathbf{x}, \mathbf{x}_{\mathrm{i}}^{n n}\right)\right) / 2$.

Step 3. Calculate the ERP-induced inner product of the $k$-nearest neighbors of sample $\mathbf{x}$,

$\mathbf{K}_{\text {erp }}(i, j)=\left\langle\mathbf{x}_{j}^{n n}, \mathbf{x}_{i}^{n n}\right\rangle_{\operatorname{erp}}$.

Step 4. Calculate the self-inner product of the sample $\mathbf{x},\langle\mathbf{x}, \mathbf{x}\rangle_{\text {erp }}$.

Step 5. Calculate $\mathbf{G}_{\text {erp }}=\mathbf{K}_{\text {erp }}+\langle\mathbf{x}, \mathbf{x}\rangle_{\operatorname{erp}} \mathbf{1}_{k k}-\mathbf{1}_{k} \mathbf{k}_{\text {erp }}^{\mathrm{T}}-\mathbf{k}_{\text {erp }} \mathbf{1}_{k}^{\mathrm{T}}$.

Step 6. Calculate $\mathbf{w}$ by solving $\left[\mathbf{G}_{\text {erp }}+\eta \mathbf{I}_{k} \operatorname{tr}\left(\mathbf{G}_{\text {erp }}\right) / k\right] \mathbf{w}=\mathbf{1}_{k}$.

Step 7. Assign the class label $\omega_{j_{\max }}=\arg \max _{\omega_{j}}\left(\sum_{y_{i}^{n n}=\omega_{j}} w_{i}\right)$ to the sample $\mathbf{x}$.

Algorithm 1: EDKC.

The combination of similarity measure with advanced KNN classifiers is expected to be more promising. So, by using DFWKNN, we intend to develop a more effective classifier, the ERP-based DFWKNN classifier (EDKC), for pulse waveform classification. Utilizing the metric property of the ERP distance, we first develop an ERP-induced inner product, and then embed this novel inner product into DFWKNN to develop the EDKC classifier.

Let $\langle\cdot, \cdot\rangle_{\text {erp }}$ denote the ERP-induced inner product. Since ERP is a metric. We can get the following heuristic deduction:

$$
\begin{aligned}
d_{\mathrm{erp}}^{2}\left(\mathbf{x}, \mathbf{x}^{\prime}\right) & =\left\langle\mathbf{x}-\mathbf{x}^{\prime}, \mathbf{x}-\mathbf{x}^{\prime}\right\rangle_{\operatorname{erp}} \\
& =\langle\mathbf{x}, \mathbf{x}\rangle_{\mathrm{erp}}+\left\langle\mathbf{x}^{\prime}, \mathbf{x}^{\prime}\right\rangle_{\operatorname{erp}}-2\left\langle\mathbf{x}, \mathbf{x}^{\prime}\right\rangle_{\mathrm{erp}}, \\
\Longrightarrow d_{\mathrm{erp}}^{2}\left(\mathbf{x}, \mathbf{x}^{\prime}\right) & =d_{\mathrm{erp}}^{2}\left(\mathbf{x}, \mathbf{x}_{0}\right)+d_{\mathrm{erp}}^{2}\left(\mathbf{x}^{\prime}, \mathbf{x}_{0}\right)-2\left\langle\mathbf{x}, \mathbf{x}^{\prime}\right\rangle_{\mathrm{erp}},
\end{aligned}
$$

where $d_{\text {erp }}\left(\mathbf{x}, \mathbf{x}^{\prime}\right)$ is the ERP distance between $\mathbf{x}$ and $\mathbf{x}^{\prime}$, and the vector $\mathbf{x}_{\mathbf{0}}$ represents a zero-length time series. Then the ERP-induced inner product of $\mathbf{x}$ and $\mathbf{x}^{\prime}$ can be defined as follows:

$$
\left\langle\mathbf{x}, \mathbf{x}^{\prime}\right\rangle_{\operatorname{erp}}=\frac{1}{2}\left(d_{\mathrm{erp}}^{2}\left(\mathbf{x}, \mathbf{x}_{0}\right)+d_{\mathrm{erp}}^{2}\left(\mathbf{x}^{\prime}, \mathbf{x}_{0}\right)-d_{\mathrm{erp}}^{2}\left(\mathbf{x}, \mathbf{x}^{\prime}\right)\right) .
$$

In (3), the element at the $i$ th row and the $j$ th column of the Gram matrix $\mathbf{G}$ is defined as $\mathbf{G}_{i j}=\left\langle\mathbf{x}-\mathbf{x}_{i}^{n n}, \mathbf{x}-\mathbf{x}_{j}^{n n}\right\rangle$, where $\langle\cdot, \cdot\rangle$ denotes the regular inner product. In EDKC, we replace the regular inner product with the ERP-induced inner product to calculate the Gram matrix $\mathbf{G}_{\text {erp }}$, which can be rewritten as follows:

$$
\mathbf{G}_{\mathrm{erp}}=\mathbf{K}_{\mathrm{erp}}+\langle\mathbf{x}, \mathbf{x}\rangle_{\mathrm{erp}} \mathbf{1}_{k k}-\mathbf{1}_{k} \mathbf{k}_{\mathrm{erp}}^{\mathrm{T}}-\mathbf{k}_{\mathrm{erp}} \mathbf{1}_{k}^{\mathrm{T}},
$$

where $\mathbf{K}_{\text {erp }}$ is a $k \times k$ matrix with the element at $i$ th row and $j$ th column $\mathbf{K}_{\text {erp }}(i, j)=\left\langle\mathbf{x}_{i}^{n n}, \mathbf{x}_{j}^{n n}\right\rangle_{\text {erp }}, \mathbf{k}_{\text {erp }}$ is a $k \times 1$ vector with the $i$ th element $\mathbf{k}_{\text {erp }}(i)=\left\langle\mathbf{x}, \mathbf{x}_{i}^{n n}\right\rangle_{\text {erp }}$, and $\mathbf{1}_{k k}$ is a $k \times k$ matrix of which each element equals 1 .

Once we obtain the Gram matrix $\mathbf{G}_{\text {erp }}$, we can directly use DFWKNN for pulse waveform classification by solving the linear system of equations defined in (4). The detailed algorithm of EDKC is shown as Algorithm 1.

3.4. The GEKC Classifier. The Gaussian RBF kernel [28] is one of the most common kernel functions used in kernel methods. Given two time series $\mathbf{x}$ and $\mathbf{x}^{\prime}$ with the same length $n$, the Gaussian RBF kernel is defined as

$$
K_{\mathrm{RBF}}\left(\mathbf{x}, \mathbf{x}^{\prime}\right)=\exp \left(-\frac{\left\|\mathbf{x}-\mathbf{x}^{\prime}\right\|_{2}^{2}}{2 \sigma^{2}}\right),
$$

where $\sigma$ is the standard deviation. The Gaussian RBF kernel requires that the time series should have the same length, and it cannot handle the problem of time axis distortion. If the length of two time series is different, resampling usually is 
TABLE 1: Data set used in our experiments.

\begin{tabular}{lcccccc}
\hline Pulse & Moderate & Smooth & Taut & Hollow & Unsmooth & Total \\
\hline Number & 800 & 550 & 800 & 160 & 160 & 2470 \\
\hline
\end{tabular}

TABLE 2: The confusion matrix of EDKC.

\begin{tabular}{ccccccc}
\hline & & \multicolumn{5}{c}{ Predicted } \\
& & $M$ & $S$ & $T$ & $H$ & $U$ \\
\hline \multirow{4}{*}{ Actual } & $M$ & $\mathbf{7 2 0}$ & 59 & 19 & 2 & 0 \\
& $S$ & 68 & $\mathbf{4 7 3}$ & 3 & 6 & 0 \\
& $T$ & 22 & 5 & $\mathbf{7 6 4}$ & 3 & 6 \\
& $H$ & 7 & 9 & 4 & $\mathbf{1 3 9}$ & 1 \\
& $U$ & 1 & 1 & 20 & 2 & $\mathbf{1 3 6}$ \\
\hline
\end{tabular}

required to normalize them to the same length before further processing. Thus Gaussian RBF kernel usually is not suitable for the classification of time series data.

Actually Gaussian RBF kernel can be regarded as an embedding of Euclidean distance in the form of Gaussian function. Motivated by the effectiveness of ERP, it is interesting to embed the ERP distance into the form of Gaussian function to derive a novel kernel function, the Gaussian ERP (GERP) kernel. By this way, we expect that the GERP kernel would be effective in addressing the local time shifting problem and be more suitable for time series classification in kernel machines. Given two time series $\mathbf{x}$ and $\mathbf{x}^{\prime}$, we define the Gaussian ERP kernel function on $X$ as

$$
K_{\mathrm{erp}}\left(\mathbf{x}, \mathbf{x}^{\prime}\right)=\exp \left(-\frac{d_{\mathrm{erp}}^{2}\left(\mathbf{x}, \mathbf{x}^{\prime}\right)}{2 \sigma^{2}}\right)
$$

where $\sigma$ is the standard deviation of the Gaussian function.

We embed the GERP kernel into KDFWKNN by constructing the kernel Gram matrix $\mathbf{G}_{\text {erp }}^{\kappa}$ defined as

$$
\mathbf{G}_{\text {erp }}^{\kappa}=\mathbf{K}_{\text {erp }}^{\kappa}+\mathbf{1}_{k k}-\mathbf{1}_{k}\left(\mathbf{k}_{\text {erp }}^{\kappa}\right)^{\mathrm{T}}-\mathbf{k}_{\text {erp }}^{\kappa} \mathbf{1}_{k}^{\mathrm{T}},
$$

where $\mathbf{K}_{\text {erp }}^{\kappa}$ is a $k \times k$ matrix with its element at ith row and $j$ th column

$$
\mathbf{K}_{\mathrm{erp}}^{\kappa}(i, j)=K_{\operatorname{erp}}\left(\mathbf{x}_{j}^{n n}, \mathbf{x}_{i}^{n n}\right),
$$

and $\mathbf{k}_{\mathrm{erp}}^{\kappa}$ is a $k \times 1$ vector with its $i$ th element

$$
\mathbf{k}_{\mathrm{erp}}^{\kappa}(i)=K_{\operatorname{erp}}\left(\mathbf{x}, \mathbf{x}_{i}^{n n}\right)
$$

Once we have obtained the kernel Gram matrix $\mathbf{G}_{\text {erp }}^{\kappa}$, we can use KDFWKNN for pulse waveform classification by solving the linear system of equations defined in (6). The details of the GEKC algorithm are shown as Algorithm 2.

\section{Experimental Results}

In order to evaluate the classification performance of EDKC and GEKC, by using the device described in Section 2.1, we construct a data set which consists of 2470 pulse waveforms
TABLE 3: The confusion matrix of GEKC.

\begin{tabular}{ccccccc}
\hline & & \multicolumn{5}{c}{ Predicted } \\
& & $M$ & $S$ & $T$ & $H$ & $U$ \\
\hline \multirow{4}{*}{ Actual } & $M$ & $\mathbf{7 3 0}$ & 54 & 15 & 1 & 0 \\
& $S$ & 61 & $\mathbf{4 7 9}$ & 4 & 6 & 0 \\
& $T$ & 16 & 2 & $\mathbf{7 7 5}$ & 1 & 6 \\
& $H$ & 7 & 7 & 2 & $\mathbf{1 4 3}$ & 1 \\
& $U$ & 0 & 1 & 19 & 1 & $\mathbf{1 3 9}$ \\
\hline
\end{tabular}

TABLe 4: Comparison of different methods for pulse waveforms classification with their accuracies achieved in recent literature.

\begin{tabular}{llrrc}
\hline \multirow{2}{*}{\begin{tabular}{l}
\multirow{2}{*}{ Mategory } \\
Methods
\end{tabular}} & \multicolumn{2}{c}{ Data set } & \multirow{2}{*}{ Accuracy } \\
\hline $\begin{array}{l}\text { Representation- } \\
\text { based }\end{array}$ & DT-M4 [22] & 372 & 3 & $92.2 \%$ \\
methods & Wavelet Network & 600 & 6 & $83 \%$ \\
& [23] & & & \\
& Artificial Neural & 63 & 3 & $73 \%$ \\
Similarity & Network [21] & 21 & 2 & $90 \%$ \\
measure-based & EDTW [19] & 1000 & 5 & $92.3 \%$ \\
methods & GEKC & 2470 & 5 & $90.36 \%$ \\
& & 2470 & 5 & $91.74 \%$ \\
\hline
\end{tabular}

of five pulse patterns, including moderate $(M)$, smooth $(S)$, taut $(T)$, hollow $(H)$, and unsmooth $(U)$. All of the data are acquired at the Harbin Binghua Hospital under the supervision of the TCPD experts. All subjects are patients in the hospital between 20 and 60 years old. Clinical data, for example, biomedical data and medical history, are also obtained for reference. For each subject, only the pulse signal of the left hand is acquired, and three experts are asked to determine the pulse pattern according to their pulse signal and the clinical data. If the diagnosis results of the experts are the same, the sample is kept in the data set, else it is abandoned. Table 1 lists the number of pulse waveforms of each pulse pattern. To the best of our knowledge, this data set is the largest one used for pulse waveform classification.

We make use of only one period from each pulse signal and normalize it to the length of 150 points. We randomly split the data set into three parts of roughly equal size and use the 3 -fold cross-validation method to assess the classification performance of each pulse waveform classification method. To reduce bias in classification performance, we adopt the average classification rate of the 10 runs of the 3 -fold crossvalidation. Using the stepwise selection strategy [26], we choose the optimal values of hyperparameters $k, \eta$, and $\sigma$ : $k=4, \eta=0.01$ for EDKC, and $k=31, \eta=0.01, \sigma=16$ for GEKC. The classification rates of the EDKC and GEKC classifiers are $90.36 \%$ and $91.74 \%$, respectively. Tables 2 and 3 list the confusion matrices of EDKC and GEKC, respectively.

To provide a comprehensive performance evaluation of the proposed methods, we compare the classification rates of EDKC and GEKC with several achieved accuracies in the recent literature $[19,21-23]$. Table 4 lists the sizes of the data set, the number of pulse waveform classes, and the achieved classification rates of several recent pulse waveform 
Input: The unclassified sample $\mathbf{x}$, the training samples $X=\left\{\mathbf{x}_{1}, \ldots, \mathbf{x}_{n}\right\}$ with the corresponding class labels

$\left\{y_{1}, \ldots, y_{n}\right\}$, the regularization parameter $\eta$, the kernel parameter $\sigma$, and the number of nearest neighbors $k$.

Output: The predicted class label $\omega_{j_{\max }}$ of the sample $\mathbf{x}$.

Step 1. Use the ERP distance to obtain the $k$-nearest neighbors $\left[\mathbf{x}_{1}^{n n}, \ldots, \mathbf{x}_{k}^{n n}\right]$ of the sample $\mathbf{x}$, and their corresponding class labels $\left[y_{1}^{n n}, \ldots, y_{k}^{n n}\right]$.

Step 2. Calculate the GERP-induced inner product between samples $\mathbf{x}$ and each of its nearest neighbors $\mathbf{k}_{\text {erp }}^{\kappa}(i)=\exp \left(-d_{\text {erp }}^{2}\left(\mathbf{x}, \mathbf{x}_{i}^{n n}\right) / 2 \sigma^{2}\right)$.

Step 3. Calculate the GERP-induced inner product of the $k$-nearest neighbors of $\mathbf{x}$ $\mathbf{K}_{\text {erp }}^{\kappa}(i, j)=\exp \left(-d_{\operatorname{erp}}^{2}\left(\mathbf{x}_{j}^{n n}, \mathbf{x}_{i}^{n n}\right) / 2 \sigma^{2}\right)$.

Step 4. Calculate $\mathbf{G}_{\text {erp }}^{\kappa}=\mathbf{K}_{\text {erp }}^{k}+\mathbf{1}_{k k}-\mathbf{1}_{k}\left(\mathbf{k}_{\text {erp }}^{\kappa}\right)^{\mathrm{T}}-\mathbf{k}_{\text {erp }}^{\kappa} \mathbf{1}_{k}^{\mathrm{T}}$.

Step 5. Calculate $\mathbf{w}$ by solving $\left[\mathbf{G}_{\text {erp }}^{\kappa}+\eta \mathbf{I}_{k} \operatorname{tr}\left(\mathbf{G}_{\text {erp }}^{\kappa}\right) / k\right] \mathbf{w}=\mathbf{1}_{k}$.

Step 6. Assign the class label $\omega_{j \max }=\arg \max _{\omega_{j}}\left(\sum_{y_{i}^{n n}=\omega_{j}} w_{i}\right)$ to the sample $\mathbf{x}$.

Algorithm 2: GEKC.

TABLE 5: The average classification rates (\%) of different methods.

\begin{tabular}{|c|c|c|c|c|c|c|c|}
\hline Pulse waveform & 1NN-Euclidean & 1NN-DTW & 1NN-ERP & Wavelet network [23] & IDTW [19] & EDKC & GEKC \\
\hline Moderate & 86.11 & 82.44 & 88.31 & 87.23 & 87.31 & 89.94 & 91.25 \\
\hline Smooth & 85.02 & 81.16 & 86.31 & 85.36 & 80.38 & 86.00 & 87.09 \\
\hline Taut & 95.76 & 87.95 & 95.10 & 89.63 & 93.15 & 95.50 & 96.88 \\
\hline Hollow & 86.75 & 82.44 & 87.56 & 85.63 & 80.44 & 86.88 & 89.38 \\
\hline Unsmooth & 84.06 & 70.81 & 84.75 & 80.63 & 89.50 & 85.00 & 86.88 \\
\hline Average & 87.36 & 83.19 & 89.79 & 87.08 & 88.90 & 90.36 & 91.74 \\
\hline
\end{tabular}

classifiers, including improved dynamic time warping (IDTW) [19], decision tree (DT-M4) [22], artificial neural network [21], and wavelet network [23]. From Table 4, one can see that GEKC achieves higher accuracy than wavelet network [23] and artificial neural network [21]. Moreover, although IDTW and DT-M4 reported somewhat higher classification rates than our methods, the size of the data set used in our experiments is much larger than those used in these two methods, and DT-M4 is only tested on a 3-class problem. In summary, compared with these approaches, EDKC and GEKC are very effective for pulse waveform classification.

To provide an objective comparison, we independently implement two pulse waveform classification methods listed in Table 4, that is, IDTW [19] and wavelet network [23], and evaluate their performance on our data set. The average classification rates of these two methods are listed in Table 5 . Besides, we also compare the proposed methods with several related classification methods, that is, nearest neighbor with Euclidean distance (1NN-Euclidean), nearest neighbor with dynamic time warping (1NN-DTW), and nearest neighbor with ERP distance (1NN-ERP). These results are also listed in Table 5. From Table 5, one can see that, our methods outperform all the other methods in term of the overall average classification accuracy.

\section{Conclusion}

By incorporating the state-of-the-art time series matching method with the advanced KNN classifiers, we develop two accurate pulse waveform classification methods, EDKC and GEKC, to address the intraclass variation and the local time shifting problems in pulse patterns. To evaluate their classification performance, we construct a data set of 2470 pulse waveforms, which may be the largest data set yet used in pulse waveform classification. The experimental results show that the proposed GEKC method achieves an average classification rate of $91.74 \%$, which is higher than or comparable with those of other state-of-the-art pulse waveform classification methods.

One potential advantage of the proposed methods is to utilize the lower bounds and the metric property of ERP for fast pulse waveform classification and indexing [29]. In our future work, we will further investigate accurate and computationally efficient ERP-based classifiers for various computerized pulse diagnosis tasks.

\section{Acknowledgments}

The paper is partially supported by the GRF fund from the HKSAR Government, the central fund from the Hong Kong Polytechnic University, the National S\&T Major project of China under Contract no. 2008ZXJ09004-035, and the NSFC/SZHK innovation funds of China under Contracts nos. 60902099, 60871033, and SG200810100003A.

\section{References}

[1] S. Z. Li, Pulse Diagnosis, Paradigm Press, 1985.

[2] H. Dickhaus and H. Heinrich, "Classifying biosignals with wavelet networks: a method for nonivasive diagnosis," IEEE 
Engineering in Medicine and Biology Magazine, vol. 15, no. 5, pp. 103-111, 1996.

[3] H. Adeli, S. Ghosh-Dastidar, and N. Dadmehr, "A waveletchaos methodology for analysis of EEGs and EEG subbands to detect seizure and epilepsy," IEEE Transactions on Biomedical Engineering, vol. 54, no. 2, pp. 205-211, 2007.

[4] H. Wang and Y. Cheng, "A quantitative system for pulse diagnosis in traditional Chinese medicine," in Proceedings of the 27th Annual International Conference of the Engineering in Medicine and Biology Society (EMBS '05), pp. 5676-5679, September 2005.

[5] S. E. Fu and S. P. Lai, "A system for pulse measurement and analysis of Chinese medicine," in Proceedings of the 11th Annual International Conference of the IEEE Engineering in Medicine and Biology Society, pp. 1695-1696, November 1989.

[6] J. Lee, J. Kim, and M. Lee, "Design of digital hardware system for pulse signals," Journal of Medical Systems, vol. 25, no. 6, pp. 385-394, 2001.

[7] W. Ran, J. I. Jae, and H. P. Sung, "Estimation of central blood pressure using radial pulse waveform," in Proceedings of the International Symposium on Information Technology Convergence (ISITC '07), pp. 250-253, November 2007.

[8] R. Leca and V. Groza, "Hypertension detection using standard pulse waveform processing," in Proceedings of IEEE Instrumentation and Measurement Technology Conference (IMTC '05), pp. 400-405, May 2005.

[9] C.-C. Tyan, S.-H. Liu, J.-Y. Chen, J.-J. Chen, and W.-M. Liang, "A novel noninvasive measurement technique for analyzing the pressure pulse waveform of the radial artery," IEEE Transactions on Biomedical Engineering, vol. 55, no. 1, pp. 288 $297,2008$.

[10] L. Xu, D. Zhang, and K. Wang, "Wavelet-based cascaded adaptive filter for removing baseline drift in pulse waveforms," IEEE Transactions on Biomedical Engineering, vol. 52, no. 11, pp. 1973-1975, 2005.

[11] C. Xia, Y. Li, J. Yan et al., "A practical approach to wrist pulse segmentation and single-period average waveform estimation," in Proceedings of the 1st International Conference on BioMedical Engineering and Informatics (BMEI '08), pp. 334338, May 2008.

[12] H. Yang, Q. Zhou, and J. Xiao, "Relationship between vascular elasticity and human pulse waveform based on FFT analysis of pulse waveform with different age," in Proceedings of the International Conference on Bioinformatics and Biomedical Engineering, pp. 1-4, 2009.

[13] Q.-L. Guo, K.-Q. Wang, D.-Y. Zhang, and N.-M. Li, "A wavelet packet based pulse waveform analysis for cholecystitis and nephrotic syndrome diagnosis," in Proceedings of the International Conference on Wavelet Analysis and Pattern Recognition (ICWAPR '08), pp. 513-517, August 2008.

[14] P.-Y. Zhang and H.-Y. Wang, "A framework for automatic time-domain characteristic parameters extraction of human pulse signals," EURASIP Journal on Advances in Signal Processing, vol. 2008, Article ID 468390, 9 pages, 2008.

[15] L. Xu, D. Zhang, K. Wang, and L. Wang, "Arrhythmic pulses detection using Lempel-Ziv complexity analysis," EURASIP Journal on Applied Signal Processing, vol. 2006, Article ID 18268, 12 pages, 2006.

[16] J.-J. Shu and Y. Sun, "Developing classification indices for Chinese pulse diagnosis," Complementary Therapies in Medicine, vol. 15, no. 3, pp. 190-198, 2007.

[17] J. Allen and A. Murray, "Comparison of three arterial pulse waveform classification techniques," Journal of Medical Engineering and Technology, vol. 20, no. 3, pp. 109-114, 1996.
[18] L. Xu, M. Q.-H. Meng, K. Wang, W. Lu, and N. Li, "Pulse images recognition using fuzzy neural network," Expert Systems with Applications, vol. 36, no. 2, pp. 3805-3811, 2009.

[19] L. Wang, K.-Q. Wang, and L.-S. Xu, "Recognizing wrist pulse waveforms with improved dynamic time warping algorithm," in Proceedings of the International Conference on Machine Learning and Cybernetics, pp. 3644-3649, August 2004.

[20] J. Lee, "The systematical analysis of oriental pulse waveform: a practical approach," Journal of Medical Systems, vol. 32, no. 1, pp. 9-15, 2008.

[21] C. Chiu, B. Liau, S. Yeh, and C. Hsu, "Artificial neural networks classification of arterial pulse waveforms in cardiovascular diseases," in Proceedings of the 4th Kuala Lumpur International Conference on Biomedical Engineering, Springer, 2008.

[22] H. Wang and P. Zhang, "A quantitative method for pulse strength classification based on decision tree," Journal of Software, vol. 4, no. 4, pp. 323-330, 2009.

[23] L. S. Xu, K. Q. Wang, and L. Wang, "Pulse waveforms classification based on wavelet network," in Proceedings of the 27th Annual International Conference of the Engineering in Medicine and Biology Society (EMBS '05), pp. 4596-4599, September 2005.

[24] B. Yi, H. V. Jagadish, and C. Faloutsos, "Efficient retrieval of similar time sequences under time warping," in Proceedings of the 14th International Conference on Data Engineering, pp. 201-208, February 1998.

[25] L. Chen and R. Ng, "On the marriage of Lp-norms and edit distance," in Proceeding of the 30th Very Large Data Bases Conference, pp. 792-801, 2004.

[26] W. Zuo, D. Zhang, and K. Wang, "On kernel differenceweighted $k$-nearest neighbor classification," Pattern Analysis and Applications, vol. 11, no. 3-4, pp. 247-257, 2008.

[27] M. R. Gupta, R. M. Gray, and R. A. Olshen, "Nonparametric supervised learning by linear interpolation with maximum entropy," IEEE Transactions on Pattern Analysis and Machine Intelligence, vol. 28, no. 5, pp. 766-781, 2006.

[28] B. Schölkopf and A. J. Smola, Learning with Kernels, MIT Press, Cambridge, Mass, USA, 2002.

[29] L. Chen, M. T. Ozsu, and V. Oria, "Robust and efficient similarity search for moving object trajectories," Tech. Rep. CS-2003-30. 